\title{
TWITTANDO FUTEBOL: BENFICA, FC PORTO E SPORTING NA LIGA NOS
}

\author{
${ }^{1}$ Célia Gouveia \\ ${ }^{2}$ Gustavo Cardoso
}

\section{Resumo}

Objetivo do estudo: O Twitter insinuou-se em todas as fendas comunicativas das sociedades contemporâneas, incluindo as definidas no contexto desportivo. Face a este panorama, é nosso propósito, a análise de 36334 posts publicados no Twitter pelos clubes da Liga NOS: Benfica, FC Porto e Sporting, no período temporal da época desportiva de 2017/2018.

Metodologia/abordagem: $\mathrm{O}$ conjunto de dados em análise foi obtido com acesso à plataforma Crimson Hexagon, e teve como objetivos mapear um conjunto de variáveis de caracterização sociográfica, identificar seguidores com poder de influência e através de uma perspetiva comparativa, proceder à análise dos principais traços geradores de envolvimento e mapear os períodos de maior fluxo.

Originalidade/Relevância: Este tipo de análise é importante para identificar a natureza plural e estratificada da filiação clubista, através das interações no Twitter, cada vez mais arraigadas na ecologia dos media.

Principais resultados: Os resultados apontam que a demografia do Twitter afeta aos clubes tende a um percentual de utilizadores jovens e urbanos - nativos digitais. Os clubes possuem uma base de seguidores com abrangência global, entre eles, utilizadores com poder de influência na rede. Constatámos, ainda, que os períodos de maior fluxo comunicacional encontram eco nos êxitos ou momentos simbólicos.

Contribuições teóricas/metodológicas: $\mathrm{O}$ artigo tem a seguinte agenda: (i) mapear um conjunto de variáveis de caracterização sociográfica; (ii) identificar seguidores com poder de influência (lead users); e, através de uma perspetiva comparada, (iii) apontar traços geradores de envolvimento e mapear os períodos que geraram maior fluxo de mensagens.

Palavras-chave: Comunicação. Twitter. Futebol.

\section{Como referenciar em APA:}

Gouveia, C., \& Cardoso, G. (2019). Twittando Futebol: Benfica, FC Porto e Sporting na Liga Nos. PODIUM Sport, Leisure And Tourism Review, 8(2), 211-229. https://doi.org/10.5585/podium.v8i2.13229

\footnotetext{
${ }^{1}$ ISCTE - Instituto Universitário de Lisboa, Centro de Investigação e Estudos de Sociologia (CIES-IUL), Lisboa, (Portugal). E-mail: E-mail: celiamargou@ gmail.com Orcid: https://orcid.org/0000-0001-5721-8922

${ }^{2}$ ISCTE - Instituto Universitário de Lisboa, Centro de Investigação e Estudos de Sociologia (CIES-IUL), Lisboa, (Portugal). E-mail: gustavo.cardoso@ iscte-iul.pt Orcid: https://orcid.org/0000-0003-2067-4718
} 
TWITTER IN FOOTBALL: BENFICA, FC PORTO E SPORTING IN PORTUGUESE LEAGUE

\begin{abstract}
Objective: Twitter insinuated itself into all the communicative slits of contemporary societies, including those defined in the sporting context. Within the scope of this panorama, it is our purpose, the analysis of 36334 posts published on Twitter by the so-called "big three" clubs of Liga NOS: Benfica, FC Porto and Sporting, in the time period of the 2017/2018 sports season.
\end{abstract}

Methodology / Approach: The data set under analysis was obtained with access to the Crimson Hexagon platform, and its objectives were to map a set of socio-graphical characterization variables, to identify followers with influence power and through a comparative perspective, to analyze the main features that generate involvement and to map the periods of greatest flow.

Originality / Relevance: This type of analysis is important to identify the plural and stratified nature of club membership through Twitter interactions increasingly rooted in media ecology.

Main results: Results show that Twitter's demographics affect to the clubs tends to a percentage of young and urban users - digital natives. Clubs have a globally-based follower base, including influential users on the network. We also found that periods of greater communication flow echo in successes or symbolic moments.

Theoretical / methodological contributions: The article has the following agenda: (i) map a set of variables of sociographic characterization; (ii) identify followers with lead power (lead users); and, from a comparative perspective, (iii) point out engendering traits and map the periods that generated the highest message flow.

Keywords: Communication. Twitter. Football. 


\section{TWEETING FÚTBOL: BENFICA, FC PORTO Y SPORTING EN LA LIGA NOS}

\section{Resumen}

Objetivo: Twitter se ha infiltrado en cada brecha comunicativa en las sociedades contemporáneas, incluidas las definidas en el contexto deportivo. Por eso, nuestro propósito es analizar 36334 publicaciones hechas en Twitter por los clubes de la Liga NOS: Benfica, FC Porto y Sporting, durante la temporada deportiva 2017/2018.

Método: El conjunto de datos bajo análisis se obtuvo con acceso a la plataforma Crimson Hexagon, y sus objetivos eran mapear un conjunto de variables de caracterización socio-gráficas, identificar seguidores con poder de influencia y, a través de una perspectiva comparativa, analizar las principales características que generan participación y mapear los períodos de mayor flujo.

Originalidad / Relevancia: Este tipo de análisis es importante para identificar la naturaleza plural y estratificada de la membresía del club a través de las interacciones de Twitter cada vez más arraigadas en la ecología de los medios.

Resultados: Los resultados muestran que la demografía de Twitter afecta a los clubes tiende a un porcentaje de usuarios jóvenes y urbanos: nativos digitales. Los clubes tienen una base de seguidores a nivel mundial, incluidos los usuarios influyentes en la red. También encontramos que los períodos de mayor flujo de comunicación resuenan en éxitos o momentos simbólicos.

Contribuciones teóricas / metodológicas: El artículo tiene la siguiente agenda: (i) mapear un conjunto de variables de caracterización sociográfica; (ii) identificar seguidores con poder de liderazgo; y, desde una perspectiva comparativa, (iii) señale rasgos engendradores y mapear los períodos que generaron el flujo de mensajes más alto.

Palabras clave: Comunicación. Twitter. Fútbol. 


\section{Introdução}

A ascensão da internet é apenas um aspecto da revolução digital, uma transformação que trouxe um crescente número de fontes de dados, que incluem áreas tão diversas como as redes sociais online (Gouveia, Lapa, e Fátima, 2018). Em Portugal, o uso do Facebook pelas comunidades desportivas tem sido intenso, ainda assim, no último ano assistimos ao incremento de plataformas alternativas como o Twitter, particularmente por grupos mais jovens (DMS, 2018). Para Rowe (2014), o Twitter insinuouse em todas as fendas comunicativas do quotidiano das sociedades contemporâneas, incluindo as definidas no contexto desportivo, sendo claramente privilegiada a relação que as comunidades desportivas estabelecem durante os eventos desportivos ao vivo (Sanderson, 2011; Price, Farrington, e Hall, 2013).

Neste campo interpretativo de inovações comunicativas, como é o caso do Twitter, o futebol extravasa para além daquilo que se disputa dentro das quatro linhas. Apresenta oportunidades, quer como canal de comunicação privilegiado na construção de relacionamentos, quer como um recurso de marketing (Bruns, Weller e Harringto, 2014), constituindo-se, por isso, em um agregador de valor para as marcas (Williams, Chinn e Suleiman, 2014). Aliás, pode afirmar-se que a plataforma actua como um fórum aberto que aborda práticas de comunicação alargadas para uma audiência global (Dolles e Söderman, 2011), e que, em última análise, podem levar ao aumento de receitas para os clubes (Kassing e Sanderson, 2010; Pegoraro, 2010).

Ora, estes fatores somados apontam para uma influência do Twitter como diretor de tráfego na web, na medida em que veio permitir ao futebol alcançar um status de transmissão e receção em tempo real (Hutchins, 2011) e, como consequência, fortalecer os vínculos com os fãs (Williams et al., 2014).

O constructo global do Twitter como uma rede interconectada permitiu aos fãs passarem a produsers (Bruns, 2008) através de novas dinâmicas de difusão, que resultam na criação de conteúdo, acção critica e pluralização de narrativas. Segundo Livingstone (1999), a par de qualquer processo de difusão ocorre sempre um processo de apropriação, ou seja, a audiência é, cada vez menos, um consumidor final, para se tornar também um produtor.

Deste modo, os fãs assumiram uma posição democratizante e disruptiva da ordem social existente
(Murthy, 2013), o que não acontecia através de meios de transmissão convencionais (Papacharissi, 2015). Emerge, assim, a ideia de que o Twitter é usado pela comunidade desportiva predominantemente como uma tecnologia de fandom (Cleland, Doidge, Millward, e Widdop, 2018).

Para boyd e Crawford (2012) o Twitter fornece um bom exemplo para a análise estatística, sobretudo, porque é fácil coletar dados e permite aos pesquisadores examinar uma ampla variedade de padrões. É, pois, neste sentido que os clubes podem tirar partido dos dados para identificar padrões através da caracterização sociográfica, uso de mecanismos de identificação em torno das relações de poder na rede, contributos importantes que podem ser utilizados como estímulos para comunicar um ideal de identidade construída através da produção simbólica (Rowe, 2004; Sanderson e Kassing, 2011).

É precisamente a partir destas noções e face à crescente base de seguidores dos clubes portugueses, que o Twitter se tornou um interessante laboratório das dinâmicas e processos coletivos afetos aos clubes. Por isso, este estudo tem como objetivos: (i) mapear um conjunto de variáveis de caracterização sociográfica; (ii) identificar seguidores com poder de influência (lead users); e, através de uma perspetiva comparada, (iii) apontar traços geradores de envolvimento e mapear os períodos que geraram maior fluxo de mensagens.

A investigação, ainda que de carácter exploratório, tem por base a análise de um conjunto de dados relativos às contas oficiais no Twitter dos clubes historicamente chamados "três grandes" da Liga NOS - Sport Lisboa e Benfica (SLB), Futebol Clube do Porto (FCP) e Sporting Clube de Portugal (SCP) ${ }^{3}$. A base de dados em análise conta com 36334 tweets publicados pelos três clubes, no período temporal entre 6 de agosto de 2017 a 13 de maio de 2018, formada com a monitorização via Crimson Hexagon.

\section{Futebol como Ritual Público}

O futebol constitui um ritual público vivenciado na cultura europeia contemporânea, através da qual emergem novas solidariedades sociais (King, 2017). Em Portugal, é desporto nacional e paixão partilhada por muitos milhões (Coelho e Tiesler, 2006). Fala-se de paixão, mas o que está em "jogo", para além do próprio jogo, é a identificação mimética entre adeptos e as suas equipas ou clubes (Marivoet, Bittencourt, Melo e Tonini, 2015).

\footnotetext{
${ }^{3}$ A ordem apresentada dos clubes teve em consideração o número de seguidores nas páginas oficiais do Twitter.
} 
Há, aliás, razões para afirmar que existe em Portugal um forte envolvimento socio afetivo em torno do futebol (Nunes, 2007). Um indicador desta importância social tem sido medido pela sua expressão mediática e conferido nas conversas quotidianas dos diversos espaços sociais (Cardoso e Telo, 2018).

No entanto, com a propagação dos meios digitais, em especial com o advento das redes sociais, construíram-se novas formas de sociabilidade em torno do tema. Surgem novas dimensões: o espaço e o tempo. A dimensão espacial com ênfase na comunicação multilateral e a dimensão do tempo com relevância na sincronização, partindo do princípio que uma sequência ininterrupta de ações e reações geralmente melhoram a qualidade das interações (Dijk, 2006). Note-se, ainda, que os desportos mediados não se restringem ao dia do jogo, fazem parte de uma construção social e, consequente, impacto na identificação do fã (Wann e James, 2018).

$\mathrm{Na}$ atual ecologia dos media, onde coabitam uma imensa profusão de redes sociais, o Twitter foi a plataforma que mais rapidamente se instalou na vida das comunidades desportivas em virtude do seu design aberto, considerado uma ferramenta multifuncional (Kassing, et al., 2004; Marwick e boyd, 2011; Rowe, 2014).

$\mathrm{O}$ Twitter manteve-se aberto a uma variedade de usos, apesar do seu significado sociocultural como prática social mediada ser contestado e moldado por diversos interesses, como afirma Van Dijck (2011). Bruns (2012) destaca a sua volatilidade, por causa da grande variedade de actores e interações envolvidas, pelo facto de ser permeável e suscetível a diferentes motivações para o consumo de conteúdos por parte das culturas adeptas, que, segundo Haugh e Watkins (2016), tendem a produzir conexões várias, sobretudo por motivações comportamentais (sociais).

Neste contexto, as redes sociais online vieram oferecer aos clubes um campo de ação social e de poder onde se inscrevem dualidades inerentes à competição desportiva e às hierarquias simbólicas que estes produzem (Kassing, et al., 2004). Condições que, como seria de esperar, vieram reforçar a tendência das organizações desportivas no controlo do fluxo de informações em seu torno (Price, Farrington e Hall, 2013).

Em suma, o Twitter constituiu-se como um espaço de múltiplos fragmentos e artefactos da vida social (Rowe, 2014), onde se conjeturam (no duplo sentido) transações e negociações da identidade clubista que são transpostos para a blogosfera (Nunes, 2007). Uma mudança que, na atual ecologia dos media, se assume como o lubrificante relacional da indústria do futebol.

Atualmente, os clubes europeus têm milhões de seguidores no Twitter. Por exemplo, só o Real Madrid CF tem cerca de 109 milhões, Manchester United FC cerca de 71 milhões e Juventus FC cerca de 36 milhões de seguidores (DMS, 2018). Sem assumir números tão expressivos, os chamados "três grandes" de Portugal - Benfica, FC Porto e Sporting assumem igualmente uma dimensão mundial quanto ao número de seguidores nas redes sociais.

Segundo Hutchins (2004), o ciberespaço é um corredor virtual entre lugares, que cria associações transnacionais fluidas e flutuantes, estruturadas entre o global e o local. Dentro deste panorama, e na similitude com as comunidades adeptas, a rede pode ser olhada como o equivalente virtual da diáspora portuguesa, comunidades que de forma peculiar entroncam num forte sentimento de pertença $\mathrm{e}$ capacidade altamente desenvolvida de cooperação, o que pode ser um indicador da presença simbólica dos três grandes portugueses, nas suas muitas filiais e delegações, espalhadas pelos cinco continentes.

Note-se, ainda, que quando os seguidores das páginas dos clubes se situam num universo culturalmente semelhante, estes assumem especial importância, permitindo estabelecer um leque de relações sociais - relações de mercado, relações de poder e relações de cooperação - que, pelo seu alcance territorial, os dota de capacidade relacional (Castells, 2013). Neste sentido, Costa (2005) defende como fator integrador a necessidade de criar mensagens dotadas de simbolismo. A expressão dos símbolos comuns, a história, os objetivos e a necessidade de pertença unem a equipa à comunidade, através dos laços identitários que fortalecem a relação dos clubes com a comunidade (Haugh e Watkins, 2016) e que permitem conjugar um espaço identitário de fãs.

Se, por um lado, na sua ascensão, as plataformas sociais possibilitaram novas condições aos utilizadores, permitindo a participação na construção, enquadramento e distribuição, por outro, apresentam aspetos perniciosos, designadamente o facto de estes utilizadores moldarem, interpretarem e reinterpretarem os conteúdos e a forma como os distribuem entre os pares (Hutchins, 2016), contribuindo para a formação de identidades alternativas. Por esta razão, Bruns (2012) realça que a postura de flexibilidade dos seguidores como "produtores" conduz a fluxos dinâmicos de informações, mas também voláteis. Em boa verdade, estes seguidores podem gerar diferentes níveis de 
influência, fomentando uma natureza desigual nas relações de poder (Bruns, 2005).

É, pois, neste duplo sentido que cumpre às organizações desportivas explorar fissuras e contradições nas relações de poder. No entanto, sem descurar $o$ facto de estarem inseridas numa determinada sociedade, não podem ser compreendidas fora das crenças, valores, estruturas, práticas e tensões. Por esta razão, Tajfel (2010) afirma que os indivíduos apoiam organizações que, por norma, são congruentes com a sua identidade social.

Williams, Heiser e Chinn (2012) advogam que à medida que os membros se identificam com uma comunidade de marca, essa organização/marca tornase mais atraente e digna de apoio. Sobretudo, porque a comunidade, a marca e a organização se ligam e autoidentificam por meio de um conjunto de valores partilhados (Underwood, Bond e Baer, 2001). Quanto mais os membros da comunidade interagem com a organização, maior é a probabilidade de se considerarem membros da organização (Williams, Heiser e Chinn, 2012).

À medida que as interações no Twitter aumentam, torna-se cada vez mais importante para as organizações desportivas proceder a análises que contextualizam os fenómenos sociais e comportamentais que se desenrolam online (Sanderson, 2010; Williams e Chinn, 2010; Hambrick, 2012).

\section{O Futebol e a Rede}

Dada a natureza exploratória do estudo, e de acordo com a revisão de literatura, foi dado especial enfoque à análise dos dados que se afiguraram como relevantes para a concretização dos objetivos do trabalho, e que visam: primeiro, analisar e mapear fatores identitários, através de um conjunto de variáveis sociodemográficas (idade, gênero sexual, país e região de residência) dos seguidores das páginas oficiais que no período em análise interagiram com as mesmas; segundo, avaliar a influência de utilizadores (lead users) através da variável que classifica os utilizadores por 'pontuação de influência'. Esta métrica assenta na percentagem de postagens que @mencionam ou \#retweetam um utilizador dentro de um determinado período de tempo (Breese, 2016). Ou seja, permite-nos quantificar os autores mais populares em torno de um tweet; em terceiro, e através de uma variável que

${ }^{4}$ A Primeira Liga-Liga NOS iniciou a época desportiva de 2017/2018 a 6 de agosto com o jogo entre o C.D. das Aves x Sporting CP e terminou a 13 de maio de 2018 compara o envolvimento total (ou seja, a soma de todos os \#retweets, @ replies e @ menções) de cada monitor, pretende-se através de uma perspetiva comparada dos três grandes clubes portugueses, apontar traços geradores de envolvimento dos mesmos com os seguidores e mapear os períodos que geraram maior fluxo de mensagens.

Como referido, os dados estatísticos apresentados quedam-se ao espaço de observação das páginas oficiais dos três clubes em Portugal com mais seguidores no Twitter - Sport Lisboa e Benfica @SLBenfica; Futebol Clube do Porto - @FCPorto; Sporting Clube de Portugal - @ Sporting_CP.

Neste sentido, e para responder aos objetivos propostos foram analisados um conjunto de dados empíricos compostos por 36334 tweets publicados entre 6 de agosto de 2017 e 13 de maio de 2018, ou seja, em toda a época desportiva 2017/2018 4 .

A observação recaiu sobre estes três clubes, primeiro, porque iniciaram a atividade no Twitter em simultâneo (fevereiro de 2009). Segundo, porque detêm a hegemonia de seguidores nas mídias sociais em Portugal, no geral e no Twitter em particular. Por fim, porque são os únicos clubes com mais de meio milhão de seguidores no contexto dos clubes portugueses.

Os dados foram coletados com recurso ao software de Análise de Redes Sociais - Crimson Hexagon $(\mathrm{CH})$, um software que usa um algoritmo desenvolvido pelo Instituto de Ciências Sociais Quantitativas da Universidade de Harvard (Breese, 2016) e é usado pelo MediaLab do ISCTE-IUL, onde esta pesquisa foi realizada.

$\mathrm{O} \mathrm{CH}$ oferece a vantagem de ser um produto certificado pelo Twitter, coleta e indexa o Firehose completo do Twitter, ou seja, todos os Tweets públicos da Twittosfera (em qualquer idioma ou local) (Jamal et al., 2015). Por sua vez, a plataforma CH Foresight permite ao investigador configurar parâmetros para coletar dados, usando "monitores" que agem como interface do utilizador na coleta dos dados através do treino do algoritmo. Desta forma, o número de tweets em análise representa todo o corpus de tweets públicos, independentemente de qualquer variação de \#hashtag usada (Breese, 2016).

\section{Twittar Futebol em Portugal}

O'Hallarn et al. (2018) consideram que o futebol supõe uma multiplicidade de articulações sociais, por

com o jogo entre o C.S. Marítimo x Sporting CP (http://ligaportugal.pt/pt/homepage/). 
conseguinte, cada clube deve elaborar um plano de comunicação de acordo com o perfil dos seus seguidores. É, por isso, fundamental conhecer os seguidores para competir comercialmente num cenário mundial (Giulianotti e Robertson, 2004), o que implica a utilização de métricas no sentido de observar as suas dinâmicas, bem como o volume de dados que produzem (Billings, 2014; Breese, 2016).
Um dos indicadores da popularidade de uma página oficial está no número de seguidores (Cha, Haddadi, Benevenuto e Gummadi, 2010). No início da temporada 2017/2018, os três clubes em análise tinham as suas contas ativas e atraiam um número significativo de seguidores, números que foram aumentando ao longo da temporada, conforme se pode observar (Tabela 1).

Tabela 1 - Seguidores no Twitter entre 2017-08-6 e 2018-05-13

\begin{tabular}{cccc}
\hline & $2017-08-6$ & $2018-05-13$ & Aumento de seguidores \\
\hline SLB & 1063932 & 1192925 & $11 \%$ \\
\hline FCP & 917244 & 1072692 & $16 \%$ \\
\hline SCP & 618944 & 699393 & $12 \%$ \\
\hline
\end{tabular}

São vários os trabalhos de investigação que analisam a correlação entre a cultura de fandom e a variável idade, ainda que com observações diversas. Murrell e Dietz (1992) admitem que a cultura de fandom se correlaciona positivamente com a variável idade. Já Locke et al. (2012) concordam que indivíduos mais jovens tendem a demonstrar mais envolvimento. Por seu lado, Westerbeek (2000) e Yoh, Pai, e Pedersen (2009) consideram que fãs de diferentes faixas etárias tendem a expressar-se de forma diferente dentro do fandom.

Neste âmbito, a amostra ${ }^{5}$ (conforme Figura 1) mostra que o grupo etário mais representativo dos seguidores dos "três grandes" constitui um segmento particularmente relevante, situando-se no intervalo com idades iguais ou inferiores a 17 anos (70\%). Salienta-se, assim, a expressão adolescente dos seguidores das páginas oficiais dos clubes no Twitter.

O segundo grupo etário mais representativo é composto pelo grupo estatístico dos seguidores com idades iguais ou acima dos 35 anos (24\%). No grupo etário entre os 18-24 anos, a percentagem de seguidores é relativamente baixa $(4 \%)$. Finalmente, o grupo que menor peso detém na amostra (2\%) é o grupo etário dos 25 a 34 anos. No seu conjunto, salienta-se que a representatividade dos escalões etários assume um padrão comum aos três clubes.

Figura 1 - A repartição por escalões etários ${ }^{6}$

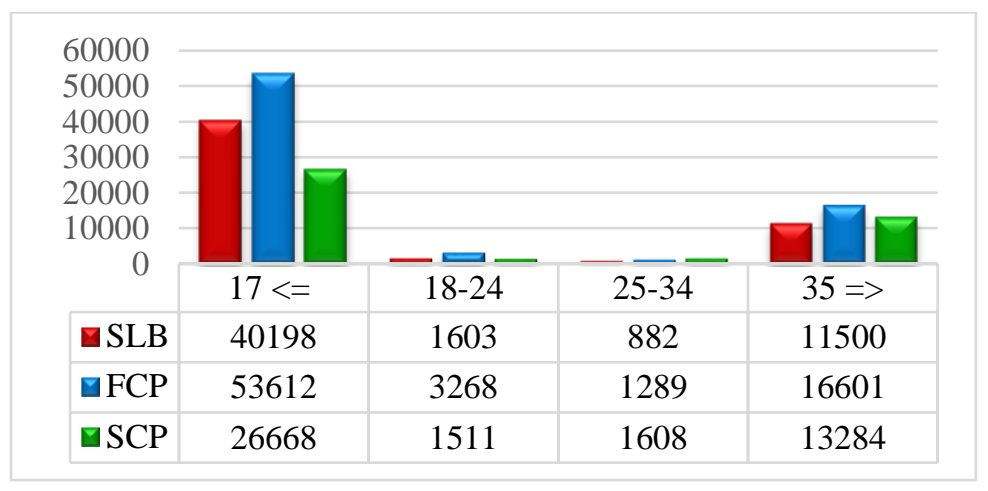

Segundo Wann e James (2018), a variável gênero é provavelmente um dos atributos sociais mais associado ao fandom desportivo. Neste sentido, e após

\footnotetext{
${ }^{5} \mathrm{O}$ Crimson Hexagon fornece uma série de informações sociodemográficas que permite a clivagem por idade, gênero sexual e em termos geográficos (região/cidade e país). Deste modo, permitiu-nos mapear perfis dos seguidores das páginas oficiais dos clubes no Twitter, durante a época 2017/2018.
}

a análise dos dados, percebemos que a distribuição segmentada dos três clubes pelo gênero sexual, resulta numa polarização, como representado na Figura $2^{7}$. 
No caso do FCP, a amostra apresenta-se equilibrada entre gêneros; no SLB, nota-se uma ligeira superioridade da representação dos homens sobre as mulheres; e no SCP, uma significativa desproporcionalidade favorável aos homens. Segundo Nunes (2007), esta polarização de gênero é, sem dúvida, um dos aspetos mais estruturantes do perfil do simpatizante de clube em Portugal.

Figura 2 - Distribuição por gênero nos três clubes

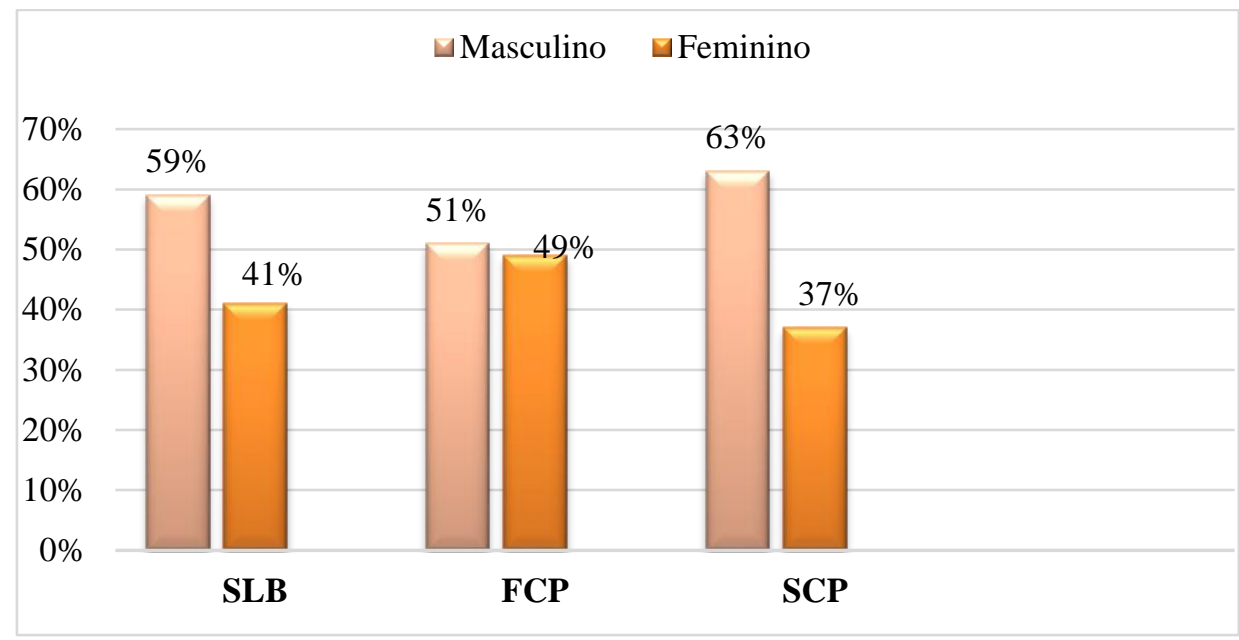

Nos últimos anos, assistimos ao rápido crescimento da chamada "Geoweb", impulsionada principalmente pela proliferação e adoção social de serviços baseados na localização e mapeamento (Barreneche, 2012). Do ponto de vista dos sistemas de informações geográficos convencionais (SIGs), a tendência de acesso às mídias sociais baseada na localização ${ }^{8}$, por um número significativo de indivíduos em todo o mundo, ainda que pouco explorado, promete ser um campo de possibilidades na análise sociogeográfica (Lee e Sumiya, 2010).

Uma das especificidades destes dados, é a possibilidade do seu uso para melhorar o sentido de conexão e construir um terreno comum de colaboração (Wilken, 2012), compreensão e mapeamento social das dinâmicas da rede (Yardi e boyd, 2010). Neste campo, acresce ainda, o potencial valor comercial dos dados, em boa verdade, o que Barreneche (2012) define como a mercantilização do espaço social, com resultados a aplicar em campanhas de marketing dirigidas e com reconhecimento local (Cheng, Caverlee e Lee, 2010).
Neste sentido, e considerando a análise do Quadro 2, da distribuição geográfica dos seguidores das páginas oficiais dos clubes no Twitter por país, verificou-se uma dimensão verdadeiramente internacional dos três clubes. Na verdade, tão ou mais importante quanto sublinhar os países mais representados, é de notar a sua dimensão global.

As variações encontradas quanto à repartição por país, entre os três clubes, são similares, não se verificando alguma supremacia expectável, relacionada com a participação nas competições da UEFA - Champions League do FCP e do SLB nas últimas temporadas.

É indispensável ressalvar, da análise dos dados apresentados, que mais de $77 \%$ dos seguidores dos três clubes estão em território português. Cerca de $18 \%$ concentram-se em dez países, entre eles, por razões históricas/e ou de similitude, países de língua oficial portuguesa, como o Brasil e Angola. Salientase, por fim, que o valor percentual restante (outros) cobre o somatório de outras localizações dispersas pelo globo.
${ }^{8}$ De acordo, com o software de análise Crimson Hexagon, só $1 \%$ dos post publicados são identificadas pelas coordenadas exatas de latitude e longitude (geotags). Assim, a localização dos restantes $99 \%$ posts têm por base em atributos conseguidos por estimativa e exibidos por publicações semelhantes às geotags, como por exemplo, informação inserida em campos predeterminados nas configurações do Twitter (Wilken, 2012). 
Tabela 2 - Distribuição geográfica dos seguidores por país

\begin{tabular}{|c|c|c|}
\hline País & Posts & \% do total \\
\hline \multicolumn{3}{|c|}{ Sport Lisboa Benfica (881876 posts identificados) } \\
\hline Portugal & 687184 & $77,92 \%$ \\
\hline Reino Unido & 33264 & $3,77 \%$ \\
\hline E. U. A. & 29693 & $3,37 \%$ \\
\hline Brasil & 27723 & $3,14 \%$ \\
\hline França & 19223 & $2,18 \%$ \\
\hline México & 13954 & $1,58 \%$ \\
\hline Alemanha & 12129 & $1,38 \%$ \\
\hline Espanha & 8423 & $0,96 \%$ \\
\hline Indonésia & 5141 & $0,58 \%$ \\
\hline Angola & 4302 & $0,49 \%$ \\
\hline Outros & 40840 & $4,63 \%$ \\
\hline \multicolumn{3}{|c|}{ Futebol Clube Porto (1352734 posts identificados) } \\
\hline Portugal & 1038047 & $76,74 \%$ \\
\hline Turquia & 37881 & $2,80 \%$ \\
\hline Reino Unido & 36498 & $2,70 \%$ \\
\hline E. U. A. & 35879 & $2,65 \%$ \\
\hline Brasil & 29981 & $2,22 \%$ \\
\hline França & 29941 & $2,21 \%$ \\
\hline Espanha & 29735 & $2,20 \%$ \\
\hline México & 25372 & $1,88 \%$ \\
\hline Indonésia & 11922 & $0,88 \%$ \\
\hline Angola & 8857 & $0,65 \%$ \\
\hline Outros & 68621 & $5,07 \%$ \\
\hline \multicolumn{3}{|c|}{ Sporting Clube Portugal ( 852249 posts identificados) } \\
\hline Portugal & 655247 & $\mathbf{7 6 , 8 8 \%}$ \\
\hline Reino Unido & 43618 & $5,12 \%$ \\
\hline E. U. A. & 30439 & $3,57 \%$ \\
\hline Brasil & 26554 & $3,12 \%$ \\
\hline Espanha & 17291 & $2,03 \%$ \\
\hline França & 14865 & $1,74 \%$ \\
\hline Angola & 6349 & $0,74 \%$ \\
\hline Indonésia & 5831 & $0,68 \%$ \\
\hline Argentina & 4858 & $0,57 \%$ \\
\hline Itália & 4394 & $0,52 \%$ \\
\hline Outros & 42803 & $\mathbf{5 , 0 3 \%}$ \\
\hline
\end{tabular}

Considerando agora a análise da distribuição geográfica dos seguidores por região/cidade (Tabela 3 ), verifica-se que os "três grandes" apresentam uma maior concentração nas cidades onde estão sedeados, o que the confere um perfil de utilizador urbano, condição que acentua os traços urbanos da sua identidade.

Assim, verifica-se uma maior presença dos seguidores do FCP na cidade do Porto. Já no caso do SLB e SCP, essa presença verifica-se em Lisboa. Ora, aqui está um dado que pode ser entendido ao arrepio do frequentemente citado "espírito bairrista", o que não significa, porém, o acantonamento dos clubes numa só cidade ou região. Repare-se, por exemplo, que a base de seguidores do SCP cobre todo o território nacional, e é, inclusive, o único clube com representação a sul, na cidade de Faro. A Península de Setúbal é a região mais a sul do território nacional com representação dos seguidores do FCP e do SLB.

São também o SLB e o FCP que apresentam um perfil de concentração regional que vai alémfronteiras. Os dados parecem, em parte, conferir uma penetração através da globalização e do posicionamento assente nas relações com a diáspora e no reforço dos laços afetivos entre a comunidade migrante e a pátria ancestral, como esferas de resistência ao "local" dentro do "global", sobretudo, no caso do SLB (Île-de-France, França e NordrheinWestfalen, Alemanha, regiões de destino da emigração portuguesa). No caso do FCP, esta 
representação flutua dinamicamente além das como o Distrito Federal - México e Região de fronteiras regionais da Europa, estende-se por regiões, Marmara -Turquia.

Tabela 3 - Distribuição geográfica dos seguidores por região/cidade

\begin{tabular}{|c|c|c|c|}
\hline Região & Cidade & Posts & $\%$ do total \\
\hline \multicolumn{4}{|c|}{ Sport Lisboa Benfica (486473 posts identificados) } \\
\hline Lisboa & Lisboa & 171277 & $35,21 \%$ \\
\hline Porto & Porto & 70683 & $14,53 \%$ \\
\hline Braga & Braga & 24960 & $5,13 \%$ \\
\hline Coimbra & Coimbra & 23450 & $4,82 \%$ \\
\hline Leiria & Leiria & 22696 & $4,67 \%$ \\
\hline Aveiro & Aveiro & 19073 & $3,92 \%$ \\
\hline Viseu & Viseu & 13272 & $2,73 \%$ \\
\hline Île-de-France (França) & Paris & 12419 & $2,55 \%$ \\
\hline Nordrhein-Westfalen (Alemanha) & Colonia & 7138 & $1,47 \%$ \\
\hline Setúbal & Setúbal & 7049 & $1,45 \%$ \\
\hline- & Outros & 114456 & $23,52 \%$ \\
\hline \multicolumn{4}{|c|}{ Futebol Clube Porto (839123 posts identificados) } \\
\hline & Porto & 442015 & $52,68 \%$ \\
\hline Porto & Lisboa & 54053 & $6,44 \%$ \\
\hline Lisboa & Braga & 53519 & $6,38 \%$ \\
\hline Braga & Aveiro & 26940 & $3,21 \%$ \\
\hline Aveiro & Istanbul & 18962 & $2,26 \%$ \\
\hline Região de Marmara (Turquia) & Setúbal & 16763 & $2,00 \%$ \\
\hline Setúbal & Coimbra & 13153 & $1,57 \%$ \\
\hline Coimbra & Cidade do & & \\
\hline Distrito Federal (México) & México & 12009 & $1,43 \%$ \\
\hline Île-de-France (França) & Paris & 11782 & $1,40 \%$ \\
\hline Viseu & Viseu & 9787 & $1,17 \%$ \\
\hline- & Outros & 180140 & $21,46 \%$ \\
\hline \multicolumn{4}{|c|}{ Sporting Clube Portugal (472228 posts identificados) } \\
\hline & Lisboa & 171872 & $36,40 \%$ \\
\hline $\begin{array}{l}\text { Porto } \\
\text { Poud }\end{array}$ & Porto & 41289 & $8,74 \%$ \\
\hline Braga & Braga & 30520 & $6,46 \%$ \\
\hline Coimbra & Coimbra & 26593 & $5,63 \%$ \\
\hline Aveiro & Aveiro & 17844 & $3,78 \%$ \\
\hline Viseu & Viseu & 16327 & $3,46 \%$ \\
\hline Santarém & Santarém & 14621 & $3,10 \%$ \\
\hline Leiria & Leiria & 13354 & $2,83 \%$ \\
\hline Setúbal & Setúbal & 11750 & $2,49 \%$ \\
\hline Faro & Faro & 7375 & $1,56 \%$ \\
\hline- & Outros & 120683 & $25,55 \%$ \\
\hline
\end{tabular}

Segundo Leen et al. (2018), plataformas como o Twitter constituem cenários de desafio do ponto de vista da abordagem na tomada de decisão em grupo, pois envolvem um grande número de utilizadores provenientes de diferentes origens e/ou com diferentes níveis de conhecimento e, porventura, capacidade de influenciar.

Estes utilizadores, "lead users" ou os influenciadores como são designados em Portugal, são "sujeitos raros" como assinala Hambrick (2012), porém, de extrema importância face às forças globalizantes que têm conduzido o futebol (Raaij, 1997).

Não é de estranhar, por isso, que muitas das contas de Twitter com mais seguidores pertencem a atletas de alta competição (Clavio e Kian, 2010).

Os dados analisados (Tabela 4) identificam os "lead users" das páginas oficiais dos clubes e sugerem uma classificação por categorias: numa primeira categoria, como era expectável, aparecem as contas oficiais dos respetivos clubes. Convém ressalvar que, 
contrariamente à arena desportiva offline, na arena desportiva da blogosfera os clubes contribuem mutuamente na orientação dos fluxos de informação e simultaneamente no processo de influência. Há, todavia, que ressaltar o facto da conta oficial do FCP não ter os clubes adversários/rivais diretos (SLB e SCP) como influenciadores, contrariamente às páginas do SLB e do SCP que seguem um padrão. Numa segunda categoria, encontramos páginas oficiais de atletas, com uma perceção mais acentuada no $\mathrm{FCP}$, com um número significativo (5 para 10) entre atletas e treinador. Numa terceira categoria, encontramos o perfil de fã, com particular destaque para o SLB (4 para 10). Finalmente, numa quarta categoria, aparecem as páginas oficiais das modalidades, que, no caso do SCP, contribuíram com uma representativa pontuação de influência.

Verificámos, ainda, que a página oficial do SLB é a única que apresenta, neste ranking, e com uma pontuação de influência expressiva a LPFP @ ligaportugal. Por fim, é possível identificar patrocinadores no top 10, porém, apenas no FCP @NBFootball (New Balance - a marca norte-americana que equipa o FCP); assim, como o principal Broadcast da Liga NOS - @SPORTTVPortugal.

Tabela 4 - Top Dez dos utilizadores mais influentes "lead users" nas páginas oficiais dos três clubes Top dez dos utilizadores mais influentes "lead users" na página oficial do SLB

\begin{tabular}{|c|c|c|c|c|}
\hline Autor & Tweets & Following & Followers & $\begin{array}{l}\text { Pontuação de } \\
\text { Influência }\end{array}$ \\
\hline @SLBenfica & 74828 & 192 & 1193104 & 14.6 \\
\hline @Raul_Jimenez9 & 3571 & 406 & 2997668 & 0.5 \\
\hline @ SadBoyyyB & 52645 & 330 & 698 & 0.3 \\
\hline @FCPorto & 45399 & 59 & 1064781 & 0.3 \\
\hline @ Sporting_CP & 80064 & 238 & 698881 & 0.3 \\
\hline$@$ SLBUltras & 241 & 3 & 7220 & 0.2 \\
\hline @ ligaportugal & 12455 & 220 & 332420 & 0.2 \\
\hline @benfica_36 & 20367 & 775 & 834 & 0.2 \\
\hline$@$ martam1904 & 56816 & 303 & 3342 & 0.1 \\
\hline @ ritapmestre & 19498 & 1600 & 3238 & 0.1 \\
\hline \multicolumn{5}{|c|}{ Top dez dos utilizadores mais influentes "lead users" na página oficial do FCP } \\
\hline Autor & Tweets & Following & Followers & $\begin{array}{c}\text { Pontuação de } \\
\text { Influência } \\
\end{array}$ \\
\hline @FCPorto & 44687 & 60 & 1073012 & 21.5 \\
\hline @IkerCasillas & 3267 & 1771 & 8359508 & 1.0 \\
\hline @DragaoRuben & 17441 & 89 & 13919 & 0.5 \\
\hline @HHerreramex & 89 & 111 & 611649 & 0.5 \\
\hline @DalotDiogo & 3547 & 99 & 24741 & 0.4 \\
\hline @CoachConceicao & 66 & 41 & 37749 & 0.4 \\
\hline @ ricbpereira & 24 & 154 & 7986 & 0.4 \\
\hline @ NBFootball & 4898 & 575 & 314976 & 0.3 \\
\hline @ SPORTTVPortugal & 17235 & 323 & 381024 & 0.3 \\
\hline @ SLBenfica & 62067 & 151 & 1098600 & 0.2 \\
\hline \multicolumn{5}{|c|}{ Top dez dos utilizadores mais influentes "lead users" na página oficial do SCP } \\
\hline Autor & Tweets & Following & Followers & $\begin{array}{c}\text { Pontuação de } \\
\text { Influência }\end{array}$ \\
\hline @Sporting_CP & 80438 & 239 & 699522 & 15.2 \\
\hline @FutFemSCP & 5497 & 207 & 9007 & 0.7 \\
\hline @SCP_Voleibol & 1696 & 3 & 2850 & 0.4 \\
\hline @ Atleti & 54073 & 56 & 4002399 & 0.4 \\
\hline @ AndebolSCP & 2466 & 82 & 4080 & 0.4 \\
\hline @ SLBenfica & 74265 & 192 & 1192076 & 0.3 \\
\hline @FCPorto & 41572 & 58 & 1032036 & 0.2 \\
\hline @SCPAdeptos1906 & 37151 & 131 & 21185 & 0.2 \\
\hline$@$ Cristiano & 3229 & 99 & 73636689 & 0.2 \\
\hline$@$ SebastianCoates & 536 & 75 & 522808 & 0.2 \\
\hline
\end{tabular}


Segundo Cha et al. (2010), há três tipos de possibilidade de interação interpessoal entre utilizadores: (i) "seguir" outros utilizadores, de forma a receber os tweets; (ii) partilhar posts/tweets de outros perfis com os seus seguidores - \#retweets; e (iii) responder ou comentar tweets de outras pessoas @ menções e @ respostas.

Através destes três tipos de interação interpessoal, o Twitter permite aos seguidores tornarem-se "imersos", através de diversas práticas multifacetadas (Mahan, 2011). Estas práticas definem atitudes e podem, segundo Bruns e Moe (2014), ser entendidas como correspondentes às camadas micro (@username), meso (seguidores na rede) e macro (\#hashtagged) das interações com os utilizadores.

Dentro do padrão que constitui a camada meso do processo de comunicação, é fácil encontrar seguidores para quem receber conteúdo é um fim em si mesmo (Honeycutt e Herring, 2009). Porém, para os clubes cujas mensagens têm como objetivo atingir milhares de seguidores, é inequívoco, que a atividade comunicativa deve ser complementada pelo uso de agregadores: \#hashtags (palavras-chave simples precedidas pelo símbolo '\#').

Note-se que as hashtag elevam a comunicação da camada meso para a macro. Esta evidência faz-nos crer que as camadas não existem isoladamente, os movimentos entre as camadas meso e macro são comuns, ainda que o mecanismo mais importante para a transição entre as três camadas sejam as práticas de retweeting, esta convenção constitui um mecanismo no qual está inerente a projeção de um tweet através dos limites das camadas (Bruns e Moe, 2014). O retweet é, por isso, a chave para a amplificação da mensagem (boyd, Golder e Lotan, 2010) é uma métrica indispensável para conhecer o valor percebido de um tweet (Kaushik, 2010), ao mesmo tempo, que projeta as práticas das contas dos clubes para a índole macro.

Por seu lado, a camada micro concentra-se nos processos comunicativos apoiados por construções sociotécnicas: @menções e @ respostas (Honeycutt e Herring, 2009; D'heer e Verdegem, 2015). Embora estas construções tenham subjacente a intenção de abordar vários utilizadores, salienta-se que a infraestrutura do Twitter torna implícito o estreitamento do foco comunicativo das mesmas. Por isso, Meraz e Papacharissi (2013) referem-se às práticas associadas a estas convenções como "marcadores de endereços".

Durante o período considerado, foram coletados dados (Figura 3) que incluem métricas quantitativas referentes aos 'posts/tweets', '\#retweets', '@respostas', '@menções', que permitem o escrutínio de valores associados ao envolvimento dos três clubes com os seguidores.

Figura 3. Métricas que permitem calcular o envolvimento

\begin{tabular}{|c|c|c|c|}
\hline 2500000 & & & \\
\hline \multicolumn{4}{|l|}{2000000} \\
\hline \multicolumn{4}{|l|}{1500000} \\
\hline \multicolumn{4}{|l|}{1000000} \\
\hline \multicolumn{4}{|l|}{500000} \\
\hline 0 & $\widehat{\text { SLB }}$ & $\overline{F C P}$ & SCP \\
\hline$\square$ Posts/tweets & 15715 & 9117 & 11502 \\
\hline$\square$ Retweets & 772138 & 1293041 & 821141 \\
\hline$\square$ Respostas & 31737 & 30251 & 22607 \\
\hline$\square$ Menções & 549208 & 730844 & 563596 \\
\hline$\square$ Total de Envolvimento & 1353083 & 2054136 & 1407344 \\
\hline
\end{tabular}

Centremos, agora, a nossa análise nos resultados, aferidos através destas métricas, como ilustrado pela Figura 3. Podemos constatar que existe um considerável diferencial entre o FCP, de um lado, e SLB e SCP, por outro, resultante do efeito dos processos de interação. É certo que os diferenciais encurtam para o SCP, mas não desaparecem.

Se, por um lado, o FCP foi o clube que menos tweets/posts publicou, por outro, foi o clube que alcançou o maior volume de retweets. Podemos 
encontrar nesta prática, um indicador de uma tentativa consciente de gerar e manter o "ruído" em torno da @FCPorto, um fenómeno conjuntural, que posiciona os seguidores como parte de um "círculo interno" que os conecta a outros fãs e incentiva à participação (Bruns, Weller e Harringto, 2014).

Para Dann (2010), os retweets aumentam a identificação do fã e fornecem um endosso que implica adicionar valor. Por esta razão, boyd, Golder, \& Lotan (2010) defendem que a prática do retweets contribui para uma ecologia conversacional composta de interação pública, um conjunto de vozes com sentido emocional.

Segundo Bruns e Moe (2014), uma comunicação de camada meso, ou seja, um processo comunicativo mais amplo, cujas mensagens atingem algumas centenas ou milhares de seguidores, em média, constitui a grande maioria da atividade comunicativa diária no Twitter.

Neste sentido, o @FCPorto registou, igualmente, o número mais elevado de 'menções', práticas que evidenciam tentativas de iniciar uma conversa com outros utilizadores. Apesar das 'menções' terem visibilidade para além dos utilizadores ativamente envolvidos, o seu foco está nesses utilizadores, indicando, sobretudo, uma intenção subjacente do foco comunicativo que a infraestrutura do Twitter torna como implícito.

Este conjunto de diferenciais, associados ao envolvimento, encurtam para o SCP, mas não desaparecem, embora o @Sporting_CP tenha publicado mais que o @FCPorto. Em contraste com esta disposição, encontramos o @SLBenfica, que sendo o clube que mais twittou, porém, foi o clube que menor envolvimento alcançou (menos 'retweets' e 'menções'), uma evidência que sugere uma comunicação assente no nível da camada micro, quer isso dizer, mais íntima. Mas os dados apontam um predomínio do @SLBenfica, como gerador de um maior número de 'respostas'. Um indicador de um tipo de comunicação, que, para Bruns e Moe (2014), constitui o nível micro da estrutura de camadas do sistema comunicativo do Twitter.

Apesar desta construção sociotécnica ter visibilidade entre os utilizadores não ativamente envolvidos, o seu foco encontra-se nesses utilizadores, logo, este é um fator que só por si não gera mais envolvimento. Não será então estranha, a diferença encontrada através da análise dos dados referentes aos níveis de envolvimento: o FCP conseguiu $43 \%$ do total do envolvimento gerado pelos três clubes, seguindo-se, em patamar percentual semelhante, o SCP com $29 \%$ e o SLB com $28 \%$.

Não deixa de ser curioso verificar (Figura 4) que o clube com o maior número de seguidores é o clube que tem o menor índice de envolvimento. Por outro lado, o SCP sendo o clube com menos seguidores (pouco mais de meio milhão), consegue um valor superior ao do clube com mais seguidores (o SLB tem quase o dobro).

Figura 4. Comparação do total de envolvimento com o número de seguidores

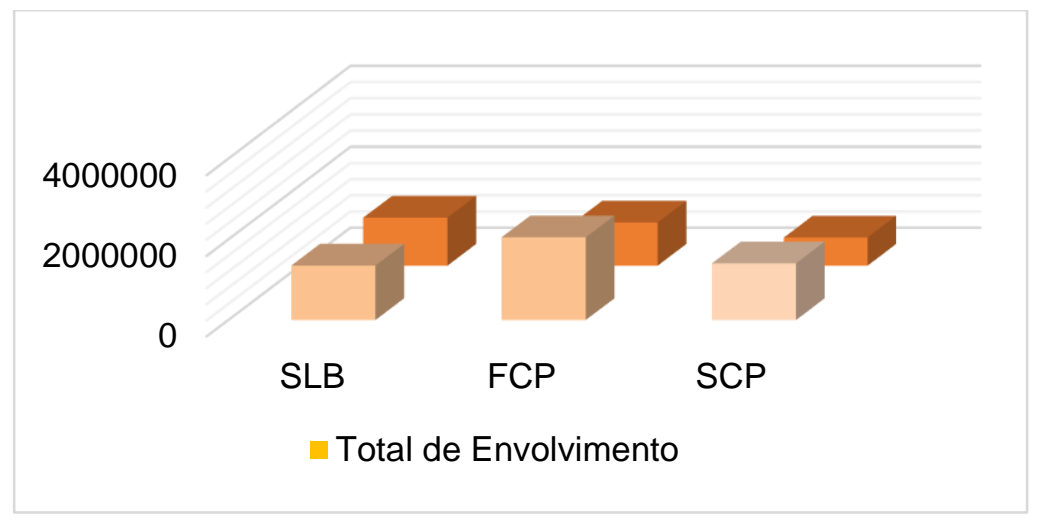

Por fim, recorre-se ao eixo de análise que nos permite traçar uma perspetiva comparativa entre os clubes, na sua relação com o envolvimento, e mapear períodos de maior fluxo, momentos entendidos como marcadores temporais importantes de comunicação a partir da intensidade das mensagens.
Durante o período considerado, o padrão de atividade quotidiana dos clubes no Twitter teve diferentes níveis de intensidade (Figura 5). Uma atividade que, segundo Bruns, Weller e Harringto (2014), pode ser caraterizada por três vias: pelo número de seguidores, desempenho desportivo e desempenho (social) dos vários canais de media do clube. 
Figura 5. Compara o envolvimento total da conta de cada clube no período em análise

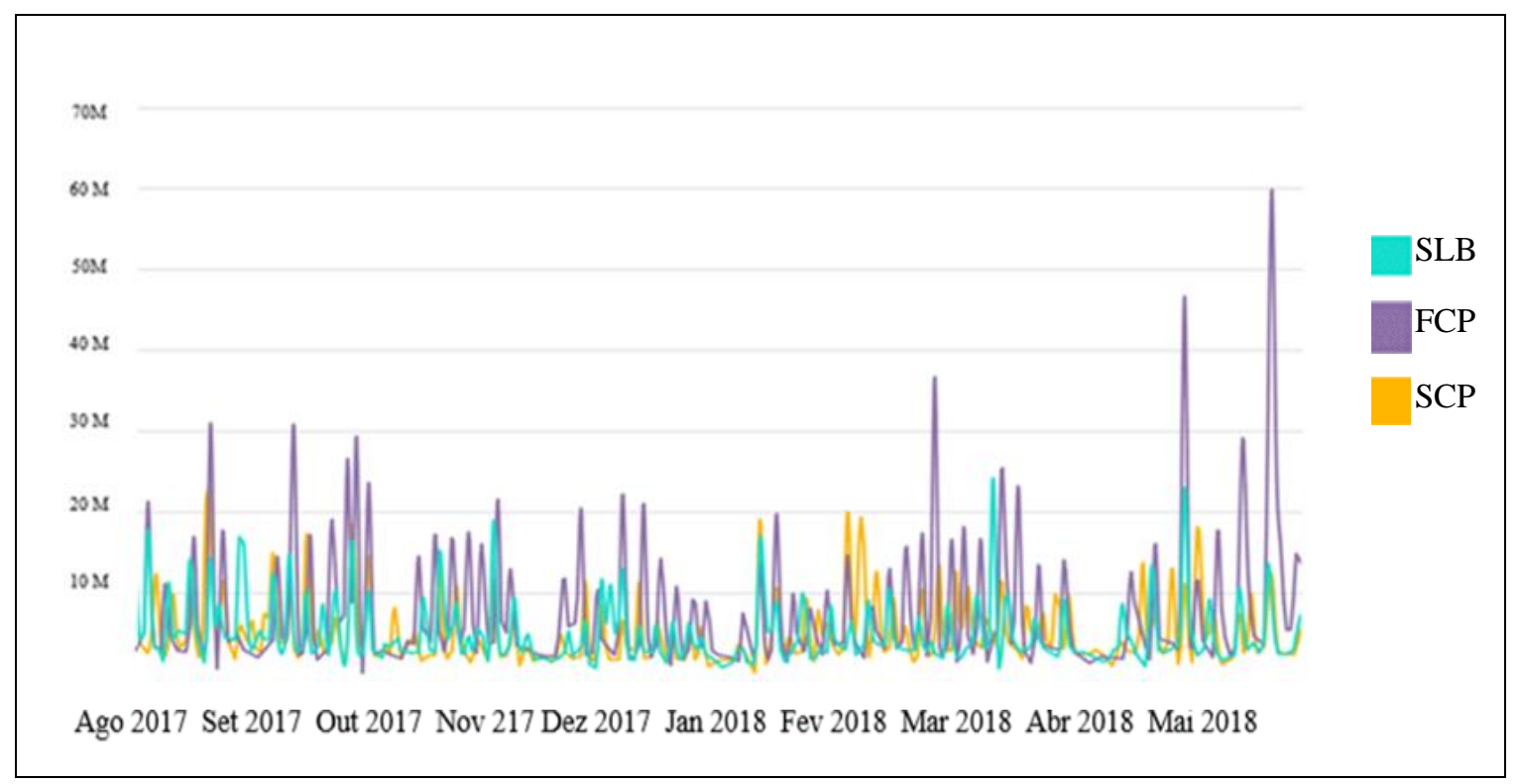

Em termos gerais, no que toca ao envolvimento, identificamos um padrão de interação que indica um fluxo de comunicação oscilante. Mormente, crescente nos períodos mais ou menos bem-sucedidos (resultados desportivos) do clube dileto. A conta do FCP foi a de maior regularidade ao longo da temporada, ainda que com um aumento significativo de interações na semana que antecedeu o términus do campeonato. Sem surpresa, com um registo máximo de envolvimento no dia $05 / 05$, dia do jogo que consagrou o FCP campeão nacional de futebol pela 28. ${ }^{\mathrm{a}}$ vez na sua história.

Nesta data, as publicações do FCP registaram um score de envolvimento de 59,765 mil, valores muitos significativos, que foram empolados pela celebração do campeonato e alavancadas pelas hashtags: \#nascidosparavencer; \#fcporto. Dignos de nota especial, são também os retweets de vários influenciadores, entre eles: @ IkerCasillas.

Em segundo lugar, com um score de envolvimento de 24,176 mil surge a conta do SLB.

Mais uma vez foi um evento singular e limitado no tempo (dia 28 de fevereiro), como que a recordar-nos a história - o aniversário do SLB - 114 anos de história. E alavancado com recurso a várias hashtag: \#114Anos;\#EPluribusUnum;

\#RaçaQuererEAmbição. Neste caso concreto, é percetível o equilíbrio entre utilizadores ativos e influenciadores no corpus do envolvimento: @fferreiraferro; @21nunogomes; @javigarcia06; @ totosalvio8 (atuais e ex-atletas do clube).

Por fim, com um score de 21,808 mil, encontramos o Sporting. Esta parcela de envolvimento registou-se no dia 23/08/2017, mais uma vez, um evento singular, o jogo que marcou o apuramento do SCP para a fase de grupos da Liga dos Campeões, um exemplo, do potencial dos clubes com as publicações e atualizações durante os jogos ao vivo. Acontecimento que contou com a participação de diferentes utilizadores, construindo um padrão comunicativo alavancado pelas hashtags: \#DiaDeSporting; \#UCL; \#FSBxSCP, e contou com influenciadores através da prática do retweet: @ChampionsLeague; @ Argentina.

\section{Conclusão: Fãs de Afetos, Clubes de Notícias}

Os resultados obtidos permitem-nos verificar que a comparação entre os três clubes é reveladora e demonstra uma série de fatores que podem influenciar as formas como o Twitter é usado para interagir com os fãs.

Como se infere da leitura dos dados, as variáveis de caracterização sociográfica, indicam que o envolvimento com as contas oficiais dos clubes é fortemente estratificado pela idade, é, de resto, assaz patente, a liderança das classes etárias mais jovens (menos de 17 anos). Conclui-se, assim, que a demografia do Twitter tende a um percentual de utilizadores jovens e urbanos - Nativos digitais.

Mesmo sabendo que as codificações futebolísticas acondicionam a heterogeneidade (Arnett e Laverie, 2000), os clubes devem, em nossa opinião, ter um olhar mais atento sobre os nativos digitais. Segundo Dingli e Seychell (2015), estes percebem o mundo com outros olhos: o que pode ser novidade para um imigrante digital, é provavelmente algo comum para 
os nativos digitais ou parte integrante das suas vidas. Nesta perspetiva, Prensky (2001) sublinha que os nativos digitais e os imigrantes digitais pertencem a dois grupos de usos distintos, havendo uma lacuna tangível entre eles, o que resulta em distinções específicas.

$\mathrm{Na}$ generalidade, os dados evidenciam uma amostra com suporte em ambos os gêneros, caso para dizermos que "o futebol já não é uma coisa só de homens" (Nunes, 2007), apesar de os homens ainda terem um percentual de representatividade mais elevado do que as mulheres nos três clubes. Dentro desta lógica, os resultados obtidos revelam um padrão: os homens mostram mais interesse no fandom desportivo ou indicam níveis mais altos de identificação com as equipas do que as mulheres (Nunes, 2007; Wann \& James, 2018).

Atualmente, a tecnologia de geolocalização e as formas emergentes do seu uso permitem monitorizar utilizadores com maior precisão, bem como, orientar os fluxos de informação (van Dijck, 2011). Neste sentido, os dados analisados permitem-nos concluir que os "três grandes" têm presença global, são marcas internacionalmente reconhecidas, cuja visibilidade no Twitter é assegurada pela atividade da sua base de fãs.

Por esta razão, as estratégias de comunicação dos clubes devem passar a ser pensadas dentro da nova economia global, como sugere Iosifidis (2015), porém, alavancadas na promoção das identidades clubistas, ainda que o foco se deva centrar na interseção dos interesses domésticos com os internacionais, dentro da lógica cultural do fandom (Bruns, Weller e Harringto, 2014).

No que se refere à dispersão geográfica dentro do espaço luso, identificamos uma base de seguidores semelhante em ternos de distribuição, ainda que não uniforme ao longo do país, com as cercanias das cidades a deterem a maior concentração. Com efeito, salientamos a importância de os clubes fomentarem sinergias entre si, os seguidores e os lugares com proveitos mútuos, como refere Barreneche (2012).

Quanto ao processo de identificação dos influenciadores, os dados sugerem uma classificação de perfis por categorias. Entre estes perfis, encontramos, sem surpresa, as páginas oficiais de atuais ou ex-atletas dos clubes, sobretudo, porque estes indivíduos representam atributos da identidade clubista e/ou nacional (Malcolm, 2012). Envolvem-se no debate, têm um papel importante na discussão, publicam e geram uma grande quantidade de retweets, isto é, segundo Kaushik (2010), demonstram a força da rede, numa lógica que confirma que o poder não é um atributo, mas sim uma relação (Castells, 2013).
Há, aliás, razões para crer que as dinâmicas dos influenciadores de países estrangeiros foram fundamentais nas ações ou eventos de maior envolvimento com as contas dos três clubes. Note-se, ainda, que os "lead users" podem representar um potente recurso de marketing para os clubes - ações sociais ou de merchandising (Hambrick e Mahoney, 2011)

Através do exercício comparativo entre os clubes, na sua relação com o envolvimento, constata-se que os clubes foram proactivos, publicaram, responderam diretamente a comentários e retweetaram. Em suma, alimentaram o debate na blogosfera, através de interconexões das camadas micro, meso e macro, determinadas pelas capacidades tecnológicas fundamentais da plataforma (Bruns e Moe, 2014). Porém, cabe notar que a conta do FCP foi a que manteve uma maior regularidade na relação com o envolvimento, notando-se um aumento significativo, durante as datas que envolveram a conquista do campeonato.

Verificamos, ainda, através do mapeamento do maior fluxo de interações, que estas ocorreram nos períodos de conquista/celebração dentro das "quatro linhas" ou em momentos históricos simbolicamente marcantes. Tal significa que as afinidades clubistas são também uma questão de rendimento desportivo, de êxitos e vitórias (Andreff \& Szymanski, 2006).

Foi, também, percetível que uma parte substancial das dinâmicas comunicativas dos clubes são de pendor informativo, o que nos leva a crer que os clubes não se envolveram de forma a disseminar conteúdo ou estimular a participação. Em suma, não demonstraram capacidade relacional (Castells 2013). Um número substancial de tweets partilha links (URLs), numa prática comunicativa que aponta no sentido da disseminação de informações, em vez do apelo ao envolvimento com os seguidores, o que indicia que os clubes não usam efetivamente o Twitter como plataforma "social" (Bruns, Weller, \& Harringto, 2014).

Cumpre reconhecer que, como qualquer pesquisa, esta apresenta algumas limitações. Assinalamos a ausência de análises qualitativas e reflexivas sobre o fenómeno, o que de certa forma, pode retirar profundidade às análises produzidas. Em contrapartida, o trabalho apresenta a captura e monitorização de um conjunto de dados relativos a um período de tempo significativo. Como já mencionado os dados estatísticos apresentados são muito relevantes, em particular os dados de caracterização sociográfica, que permitem tirar ilações sociológicas sobre a relevância do fenómeno em estudo, bem como 
as dinâmicas sociais que determinam as tendências do futebol contemporâneo. Nessa perspetiva, o trabalho fornece bases para futuras pesquisas, que possam utilizar dados evolutivos e que permitam verificar outros pressupostos.

Destacamos, ainda a importância do trabalho, primeiro pelo facto de existir em Portugal um forte envolvimento socio afetivo em torno do futebol, segundo porque, este tipo de abordagens se encontram cada vez mais arraigados na ecologia dos media, principalmente, pela lógica flexível que apresenta ao

\section{Referências}

Andreff, W. \& Szymanski, S. (2006). Handbook on the Economics of Sport. Cheltenham, UK: Edward Elgar Publishing Limited.

Arnett, D. B. \& Laverie, D. A. (2000). Fan characteristics and sporting event attendance: Examining variance in attendance. International Journal of Sports Marketing and Sponsorship, 2(3), 40-59.

Barreneche, C. (2012). Governing the geocoded world: Environmentality and the politics of location platforms. The International Journal of Research Into New Media Technologies, 18(3), 331-351.

Billings, A. (2014). Power in the Reverberation: Why Twitter Matters, But Not the Way Most Believe. Communication \& Sport, 2(2),107-112.

boyd, d., \& Crawford, K. (2012). Critical Questions for Big Data Information. Communication \& Society, 15(5), 662-679.

boyd, d., Golder, S., \& Lotan, G. (2010). Tweet, Tweet, Retweet: Conversational Aspects of Retweeting on Twitter. International Conference on System Sciences (HICSS-43). IEEE.

Breese, E. B. (2016). When marketers and academics share a research platform: The story of Crimson Hexagon. Journal of Applied Social Science, 10(1), 3-7.

Bruns, A. (2012). How long is a tweet? Mapping dynamic conversation networks on Twitter using Gawk and Gephi. Information, Communication \& Society, 15(9), 1323-1351. nível dos vários "públicos", comunicação irrestrita e grande versatilidade na transição entre as várias camadas proporcionadas pelo Twitter (Bruns e Moe, 2014). Desta forma, podem, a nosso ver, justificar-se mais trabalhos académicos de análise à natureza plural e estratificada da filiação clubista através das interações no Twitter que permitam discutir e aprofundar a relação entre os afetos dos fãs dos clubes que dinamizam a comunicação e as notícias veiculadas pelos clubes que não parecem criar tanto envolvimento quanto o pretendido.

Bruns, A. (2008). Blogs, Wikipedia, Second Life, and Beyond: From Production to Produsage. New York: Peter Lang Publishing Inc.

Bruns, A. (2005). Gatewatching: Collaborative Online News Production. New York: Peter Lang Publishing Inc.

Bruns, A. Weller, K. \& Harringto, S. (2004). Twitter and sports football fandom in emerging and established markets. In K. Weller, A. Bruns, J. Burgess, M. Mahrt, \& C. Puschmann (Eds.), Twitter and Society (pp. 263-280). New York: Peter Lang Publishing Inc.

Cardoso, G., \& Telo, D. (2018). Barómetro de Notícias 2018 - Estado das Notícias. Lisboa: Barómetro de Notícias LCC-IUL.

Castells, M. (2013). O Poder da Comunicação. Lisboa: Fundação Calouste Gulbenkian.

Cha, M., Haddadi, H., Benevenuto, F., \& Gummadi, K. P. (2010). Measuring user influence in twitter: The million follower fallacy. In fourth international AAAI conference on weblogs and social media, 10-17.

Cheng, Z., Caverlee, J., \& Lee, K. (2010). You Are Where You Tweet: A Content-Based Approach to Geo-locating Twitter Users. The 19th ACM International Conference on Information and knowled - CIKM'10. Toronto, Canada.

Clavio, G., \& Kian, E. (2010). Uses and gratifications of a retired female athlete's Twitter followers. International Journal of Sport Communication, 3(4), 485-500. 
Cleland, J., Doidge, M., Millward, P., \& Widdop, P. (2018). Collective Action and Football Fandom: A relational sociological. UK: Palgrave Macmillan.

Coelho, J. N., \& Tiesler, N. C. (2006). O Paradoxo do Jogo Português: a omnipresença do futebol e a ausência de espectadores dos estádios. Análise Social, 41(179), 519-551.

Costa, C. Ana (2005). The status and future of sport management: a delphi study. Journal of Sport Management, 19(2),117-142.

Dann, Stephen (2010). Twitter content classification. First Monday, 15(12).

D'heer, E. \& Verdegem, P. (2015). What social media data mean for audience studies: a multidimensional investigation of Twitter use during a current affairs TV programme. Information, Communication \& Society, 18(2), 221-234.

Dijk, J. A. (2006). The Network Society: Social Aspects of New Media (2 eds.). London. SAGE Publication Lda.

Dingli A. \& Seychell D. (2015). The New Digital Natives. London: Springer.

DMS - Digital Media in Sports (2018). Global Digital Football Benchmark. German: RESULT Sports. Obtido de http://digitale-sportmedien.com/category/fussball/

Dolles, H. \& Söderman, S. (2011). Sport as a Business International, Professional and Commercial Aspects. London: Palgrave Macmillan.

Giulianotti, R. \& Robertson, R. (2004). The globalization of football: A study in the glocalization of the 'serious life'. The British Journal of Sociology, 55(4), 545-568.

Gouveia, C., T. Lapa \& B. Di Fátima (2008). Benfica $v s$ Sporting: o derby visto a partir do Twitter. Observatorio (OBS*), Vol.12(2), 61-79.

Hambrick, M. E. (2012). Six degrees of information: Using social network analysis to explore the spread of information within sport social networks. International Journal of Sport Communication, 5, 16-34.

Hambrick, M. E. \& Mahoney, T. Q. (2011). 'It's incredible - trust me': Exploring the role of celebrity athletes as marketers in online social networks. Sport Management and Marketing, 10(3-4), 161- 179.

Haugh, B. R. \& Watkins, B. (2016). Tag me, tweet me if you want to reach me: An investigation into how sports fan use social media. International Journal of Sport Communication, 9(3), 278 -293.

Honeycutt, C. \& Herring, S. (2009). Beyond Microblogging: Conversation and Collaboration via Twitter. Proceedings of the Forty-Second Hawai'i International Conference on System Sciences (HICSS-42). Los Alamitos, CA: IEEE Press (1-10).

Hutchins, B. (2016). Tales of the digital sublime: Tracing the relationship between big data and professional sport. Convergence, 22(5), 494- 509.

Hutchins, B. (2011). The Acceleration of Media Sport Culture: Twitter, telepresence and online messaging. Information, Communication and Society, 14(2), 237-257.

Hutchins, B. (2004). Castells, Regional News Media and the Information Age. Journal of Media \& Cultural Studies, 18(4), 577-590.

Iosifidis, P. (2015). Global Media and Communication Policy. UK: Palgrave Macmillan.

Jamal, A., Keohane, O., Romney, D., \& Tingley, D. (2015). Anti-Americanism and AntiInterventionism in Arabic Twitter Discourses. American Political Science Association, 13(1), 55-73.

Kassing, J. W. \& Sanderson, J. (2010). FanAthlete Interaction and Twitter Tweeting Through the Giro: A Case Study. International Journal of Sport Communication, 3(1), 113-128.

Kassing, J. W., Andrew C. Billings, Robert S. Brown, Kelby K. Halone, Kristen Harrison, Bob Krizek, Lindsey J. Mean \& Paul D. Turman (2004). Communication in the Community of Sport: The Process of Enacting, (Re)Producing, Consuming, and Organizing Sport. Annals of the International Communication Association, 28(1), 373-409.

King, A. (2017). The European Ritual: Football in the New Europe. London: Routledge.

Lee, R. \& Sumiya, K. (2010). Measuring Geographical Regularities of Crowd Behaviors for 
Twitter-based Geo-social Event Detection. LBSN 10 Proceedings of the 2nd ACM SIGSPATIAL International Workshop on Location Based Social Networks, 1-10.

Leen, R., Usma, C., Antlej, K. \& Collins, P. K. (2018). Identifying, Lead Users for user-centered design (UCD) in sports product development. G Proceedings: American Bar Association. Section of International and Comparative Law, 2(6), 1- 6.

Livingstone, S. (1999), New Media, New Audiences? New Media \& Society, 1(1), 59-66.

Lock, D., Taylor, T., Funk, D. \& Darcy, S. (2012). Exploring the Development of Team Identification. Journal of Sport Management, 26(4), 283-294.

Mahan, J. E. (2011). Examining the predictors of consumer response to sport marketing via digital social media. International Journal of Sport Management and Marketing, 9(3/4), 254-267.

Marivoet, S., Bittencourt, M., Melo, V. A. \& Tonini, M. D. (2015). O que é o futebol? Lisboa: Escolar Editora.

Marwick, A. E., \& boyd, d. (2011). I tweet honestly, I tweet passionately: Twitter users, context collapse, and the imagined audience. New Media \& Society, 13(1), 114-133.

Meraz, S. \& Papacharissi, Z. (2013). Networked Gatekeeping and Networked Framing on \#Egypt. The International Journal of Press/Politics, 18(2), 138166.

Murrell, A. J., \& Dietz, B. (2011). Fan Support of Sport Teams: The Effect of a Common Group Identity. Journal of Sport and Exercise Psychology, 14(1), 28-39.

Murthy, D. (2013). Twitter: Social Communication in the Twitter Age. Cambridge, UK: Polity Press.

Nunes, J. S. (2007). Culturas Adeptas do Futebol. O espaço plural da condição adepta: práticas e identidades. Dissertação de Doutoramento em Sociologia da Cultura, Faculdade de Ciências Sociais e Humanas da Universidade Nova de Lisboa. Lisboa, Portugal.

O'Hallarn, B., Shapiro, S. L., Hambrick, M. E., Wittkower, D. E., Ridinger, L., \& Morehead, C. A.
(2018). Sport, Twitter Hashtags, and the Public Sphere: A qualitative test of the phenomenon through a curt schilling Case Study. Journal of Sport Management, 32(4), 389-400.

Papacharissi, Z. (2015). Toward New Journalism(s). Journalism Studies, 16 (1), 27-40.

Pegoraro, A. (2010). Look who's talking - athletes on Twitter: A Case study. International Journal of Sport Communication, 3, 501-514.

Prensky, M. (2001). Digital Natives, Digital Immigrants. Part I. On the Horizon, 9(5), 1-6.

Price, J., Farrington, N. \& Hall, L. (2013). Changing the game? The impact of Twitter on relationships between football clubs, supporters and the sports media. Soccer \& Society, 14(4), 446-461.

Raaij, W. F. (1997). Globalisation of marketing communication? Journal of Economic Psychology, $18,259-270$.

Rowe, D. (2014). Following the Followers: Sport Researchers' Labour Lost in the Twittersphere? Communication \& Sport, 2 (2), 117-121.

Rowe, D. (2004). Sport, Culture and the Media. UK: Open University Press

Sanderson, J. (2010). Framing Tiger's Troubles: Comparing Traditional and Social Media. International Journal of Sport Communication, 3, 438-453.

Sanderson, J. (2011). It's a whole new ballgame: How social media is changing sports. New York: Hampton Press.

Tajfel, H. (2010). Social identity and intergroup relations. New York: Cambridge University Press.

Underwood, R., Bond, E. \& Baer, R. (2001). Building service brands via social identity: Lessons from the sports marketplace. Journal of Marketing Theory and Practice, 9(1), 1-13.

Van Dijck, J. (2011). Tracing Twitter: The rise of a microblogging platform. International Journal of Media and Cultural Politics, 7(3), 333-348. 
Wann, D. L. \& James, J. D. (2018). Sport fan: The psychology and social impact of spectators (2 eds.). New York, US: Routledge.

Weller, K., Bruns, A., Burgess, J., Mahrt, M. \& Puschmann, C. (2014). Twitter and Society. New York: Peter Lang.

Westerbeek, H. (2000). The Influence of Frequency of Attendance and age on "Place"-Specific Dimensions of Service Quality at Australian Rules Football Matches. Sport Marketing Quarterly, 9 (4), $194-202$.

Wilken, R. (2012). Twitter and Geographical Location. In K. Weller, A. Bruns, J. Burgess, M. Mahrt, \& C. Puschmann (Eds.), Twitter and Society (pp. 155165). New York: Peter Lang Publishing, Inc.

Williams, J. \& Chinn, S. J. (2010). Meeting Relationship-Marketing Goals Through Social Media: A
Conceptual Model for Sport Marketers. International Journal of Sport Communication, 3(4), 422-437.

Williams, J., Chinn, S. J. \& Suleiman, J. (2014). The value of Twitter for sports fan. Journal of Direct Data and Digital Marketing Practice, 16(1), 36-50.

Williams, J., Heiser, R. \& Chinn, S. J. (2012). Social Media Posters and Lurkers: The impact on team identification and game attendance in minor league baseball. Journal of Direct, Data and Digital Marketing Practice, 13(4), 295-310.

Yardi, S. \& boyd, d. (2010). Tweeting from the town square: Measuring geographic local networks. Paper presented at the 4th International AAAI Conference on Weblogs and Social Media, (194-201). Washington, DC.

Yoh, T., Pai, H. T. \& Pedersen, P. M. (2009). The influence of socialisation agents on the fan loyalty of Korean teens. International Journal of Sport Management and Marketing, 6(4), 404-416. 